\title{
A FINITE ELEMENT METHOD FOR ELASTIC PARAMETERIZATION AND ALIGNMENT OF CORTICAL SURFACES USING SULCAL CONSTRAINTS
}

\author{
Anand A. Joshi ${ }^{1}$, David W. Shattuck ${ }^{2}$, Paul M. Thompson ${ }^{2}$, and Richard M. Leahy ${ }^{1}$ \\ ${ }^{1}$ Signal and Image Processing Institute, University of Southern California, Los Angeles, CA, USA \\ ${ }^{2}$ Laboratory of Neuro Imaging, University of California, Los Angeles, CA, USA
}

\begin{abstract}
We present a cortical surface registration method that simultaneously aligns sulcal landmarks and parameterizes two cortical surfaces. The approach is based on coregistration of cortical surface coordinate systems so that the labeled sulcal features share the same coordinates on both cortical surfaces. We model the cortex as an elastic sheet and solve the associated Cauchy-Navier equilibrium equation subject to sulcal alignment constraints. The elastic energy is computed directly with respect to the intrinsic surface geometry and discretized using a finite element method on triangular tessellations of the two surfaces. In contrast to alternative methods for cortical alignment, the method avoids the need for an intermediate flat space for sulcal landmark matching and provides a fast, accurate and inverse-consistent surface registration and parameterization for inter-subject neuroanatomical studies.
\end{abstract}

Index Terms - Cortical alignment, Elastic parameterization, Finite Elements

\section{INTRODUCTION}

Registration of surface models of the cerebral cortex has important applications in multi-subject studies of brain anatomy and function. Investigators have studied progression of disorders such as Alzheimer's disease [1], growth patterns in developing human brains [2], genetic influences [3] and the influence of medication and drug abuse on the structure and function of the brain $[4,5]$. Intersubject surface registration, or the intrasubject analysis required for longitudinal studies, presents a difficult problem due to inter-subject variability and the convoluted geometry of the cortical surface. Various landmark based and landmark free techniques have been developed for registration of two cortical surface models. Most of these methods first map the two cortical surfaces to a plane or sphere and then compute a deformation vector field that aligns sulcal landmarks with respect to their planar or spherical coordinates $[6,7,8,9,10,11,12]$.

Here we propose an FEM based elastic mapping method that avoids the use of an intermediate surface flattening step

This work is supported by NIBIB under Grant No: R01 EB002010 and NCRR under Grant No: P41 RR013642. for landmark matching. It incorporates the landmark registration into the parameterization method itself. We use the Cauchy-Navier elastic equilibrium equation for performing this matching as explained in the next section. In our earlier method $[10,12]$, surface registration was performed in two stages: (i) for each subject, parameterize the surface of each cortical hemisphere to either a unit square or disk, and (ii) find a vector field with respect to this parameterization that aligns sulcal landmarks between subjects. Registration can use linear elastic [10] or thin-plate bending energies [12] for regularizing the deformation field and covariant derivatives to make the alignment independent of the parameterization. However, in order to solve the resulting variational minimization problem, numerical derivatives were computed by resampling the brain on a uniform grid with respect to the parameterization. In addition to the computational cost of resampling and interpolation, this step results in a loss of resolution since the regular or semi-regular grid in flat space is not necessarily optimal for representing the brain in 3D space. In our new approach, we incorporate sulcal landmark alignment directly in our parameterization method and thus avoid the resampling and reparameterization step completely. This approach also has the advantage that the computation cost is relatively small and that the resulting alignment is inverse consistent [13] as will become clear from the symmetry of the cost function defined below.

\section{SURFACE REGISTRATION}

To perform cortical surface registration and parameterization with labeled sulcal curves as constraints, we model the cortical surface as an elastic sheet and solve the associated elastic equilibrium equation using an FEM. We choose the more general elastic model over a surface based harmonic mapping method $[14,15,16,17]$ because we found that the surface based harmonic mappings do not remain bijective when multiple sulcal landmark constraints are imposed on the interior of the flat parameter space. However, for the elastic model we have so far always obtained a near bijective map by adjusting the model parameters $\lambda$ and $\mu$ appropriately. The reason for this situation, intuitively, is that relative to the power of the Laplacian alone, the Cauchy-Navier elasticity operator pro- 
vides additional control over the gradient of the divergence of the surface vector field, and this indirectly controls the Jacobian of the mapping, constraining it from taking on extreme values and thereby violating the smoothness assumption.

\subsection{Mathematical Formulation}

We assume as input a pair of genus-zero, tessellated cortical surfaces extracted from a volumetric MR image [18]. Our goal is to map the surfaces of each cortical hemisphere in the two brains to the unit square such that in the flat map a set of manually delineated sulcal landmarks are aligned with respect to the flat space coordinates. Point landmarks are generated by sampling uniformly along each sulcal curve. Let $\phi=\left[\phi_{1}, \phi_{2}\right]^{T}$ be the 2D coordinates assigned to every point on a given cortical surface such that the coordinates $\phi$ satisfy the Cauchy-Navier elastic equilibrium equation with Dirichlet boundary conditions on the boundary of each cortical hemisphere, represented by the corpus callosum. We constrain the corpus callosum to lie on the boundary of the unit square mapped as a uniform speed curve. We solve the equilibrium equation in the geometry of the cortical surface using the form:

$$
\mu \Delta \phi+(\mu+\lambda) \nabla(\nabla \cdot \phi)=0 .
$$

where $\mu$ and $\lambda$ are Lamé's coefficients. The operators $\Delta$ and $\nabla$ represent the Laplace-Beltrami and covariant gradient operators, respectively, with respect to the surface geometry. The solution of this equation can be obtained variationally by minimizing the following integral on the cortical surface [19]:

$E(\phi)=\int_{S} \frac{\lambda}{4}\left(\operatorname{Tr}\left((D \phi)^{T}+D \phi\right)\right)^{2}+\frac{\mu}{2} \operatorname{Tr}\left(\left((D \phi)^{T}+D \phi\right)^{2}\right) d S$.

where $D \phi$ is the covariant derivative of the coordinate vector field $\phi$. The integral $E(\phi)$ is the total strain energy. Although the elastic equilibrium equation models only small deformations, we have found that in practice we can always compute a flat map of the cortex by setting the parameters $\mu=1$ and $\lambda=10$.

Minimizing (2) produces a flat map of each hemisphere but will not constrain the locations of the sulcal landmarks. To do this, we introduce the following constraints. Let $\phi_{S}$ and $\phi_{A}$ denote the 2D coordinates to be assigned to the subject and atlas brain hemispheres respectively. Then we define the Lagrangian cost function $C\left(\phi_{S}, \phi_{A}\right)$ as

$$
C\left(\phi_{S}, \phi_{A}\right)=E\left(\phi_{S}\right)+E\left(\phi_{A}\right)+\sigma \sum_{k \in M}\left(\phi_{S}(k)-\phi_{A}(k)\right)^{2}
$$

where $\phi_{S}(k)$ and $\phi_{A}(k)$ denote the coordinates assigned to the set of sulcal landmarks $M$, and $\sigma$ is a Lagrange multiplier. Note that we do not constrain the locations of the sulci in the flat map but simply constrain homologous landmarks in the two maps to lie at the same coordinates.

\subsection{Finite Element Formulation}

To minimize (3) on a tessellated surface we use an FEM to discretize the strain energy $E(\phi)$. Since the integrand in (3) is a tensor, it is justifiable to compute it locally at each vertex point by assigning a local coordinate system $(x, y)$ to its neighborhood. For each triangle the covariant derivative $D \phi$ in the local coordinates $x, y$ becomes the Jacobian matrix:

$$
D \phi=\left(\begin{array}{ll}
\frac{\partial \phi_{1}}{\partial x} & \frac{\partial \phi_{1}}{\partial y} \\
\frac{\partial \phi_{2}}{\partial x} & \frac{\partial \phi_{2}}{\partial y}
\end{array}\right)
$$

From (2), the strain energy $E_{i}(\phi)$ for the $i^{\text {th }}$ triangle $\Delta_{i}$ is given by:

$$
\begin{aligned}
E_{i}(\phi) & =\int_{\Delta_{i}}(2 \mu+\lambda)\left(\left(\frac{\partial \phi_{1}}{\partial x}\right)^{2}+\left(\frac{\partial \phi_{2}}{\partial y}\right)^{2}\right) \\
& +2(\mu+\lambda)\left(\frac{\partial \phi_{1}}{\partial y}\right)\left(\frac{\partial \phi_{2}}{\partial x}\right)+\mu\left(\left(\frac{\partial \phi_{1}}{\partial y}\right)^{2}+\left(\frac{\partial \phi_{2}}{\partial x}\right)^{2}\right) d S
\end{aligned}
$$

We now describe the FEM discretization of the partial derivatives with respect to the local coordinates. Let $\alpha$ be any piecewise linear real-valued scalar function defined over the surface, and $\alpha_{i}$ the function restricted to triangle $i$ with local coordinates $x, y$. Also denote the local coordinates of the three vertices as $\left(x_{1}, y_{1}\right),\left(x_{2}, y_{2}\right)$ and $\left(x_{3}, y_{3}\right)$ respectively. Since $\alpha_{i}$ is linear on the $i^{\text {th }}$ triangle, we can write,

$$
\alpha_{i}(x, y)=a_{0}^{i}+a_{1}^{i} x+a_{2}^{i} y
$$

Writing this expression at three vertices of the triangle $i$ in matrix form,

$$
\underbrace{\left(\begin{array}{lll}
1 & x_{1}^{i} & y_{1}^{i} \\
1 & x_{2}^{i} & y_{2}^{i} \\
1 & x_{3}^{i} & y_{3}^{i}
\end{array}\right)}_{D^{i}}\left(\begin{array}{l}
a_{0}^{i} \\
a_{1}^{i} \\
a_{2}^{i}
\end{array}\right)=\left(\begin{array}{l}
\alpha^{i}\left(x_{1}, y_{1}\right) \\
\alpha^{i}\left(x_{2}, y_{2}\right) \\
\alpha^{i}\left(x_{3}, y_{3}\right)
\end{array}\right)
$$

The coefficients $a_{0}^{i}, a_{1}^{i}$ and $a_{2}^{i}$ can be obtained by inverting the matrix $D^{i}$. From (6) and by inverting the matrix in (7), we obtain

$$
\begin{aligned}
\left(\begin{array}{c}
\frac{\partial \alpha^{i}}{\partial x_{i}} \\
\frac{\partial \alpha^{i}}{\partial y}
\end{array}\right) & =\left(\begin{array}{c}
a_{1}^{i} \\
a_{2}^{i}
\end{array}\right) \\
& =\frac{1}{\left|D^{i}\right|}\left(\begin{array}{ccc}
y_{2}^{i}-y_{1}^{i} & y_{3}^{i}-y_{1}^{i} & y_{1}^{i}-y_{2}^{i} \\
x_{1}^{i}-x_{2}^{i} & x_{1}^{i}-x_{3}^{i} & x_{2}^{i}-x_{1}^{i}
\end{array}\right)\left(\begin{array}{l}
\alpha^{i}\left(x_{1}, y_{1}\right) \\
\alpha^{i}\left(x_{2}, y_{2}\right) \\
\alpha^{i}\left(x_{3}, y_{3}\right)
\end{array}\right)
\end{aligned}
$$

Denote the discretization of $\frac{\partial}{\partial x}$ and $\frac{\partial}{\partial y}$ at triangle $i$ by $D_{x}^{i}$ and $D_{y}^{i}$ respectively. Also note that $\left|D^{i}\right|=2 A_{i}$ where $A_{i}$ is the area of the $i^{\text {th }}$ triangle. Then we have:

$$
\begin{aligned}
D_{x}^{i} & =\frac{1}{2 A_{i}}\left(\begin{array}{lll}
y_{2}^{i}-y_{1}^{i} & y_{3}^{i}-y_{1}^{i} & y_{1}^{i}-y_{2}^{i}
\end{array}\right) \\
D_{y}^{i} & =\frac{1}{2 A_{i}}\left(\begin{array}{lll}
x_{1}^{i}-x_{2}^{i} & x_{1}^{i}-x_{3}^{i} & x_{2}^{i}-x_{1}^{i}
\end{array}\right) .
\end{aligned}
$$


Substituting these in (5) and (3), we have

$$
\begin{aligned}
E(\phi) & =\sum_{i} \frac{1}{4 A_{i}}\left(\phi_{1}^{i} \phi_{2}^{i}\right) K\left(\begin{array}{c}
\phi_{1}^{i} \\
\phi_{2}^{i}
\end{array}\right) \\
& =\left\|\sum_{i} \frac{1}{2 \sqrt{A_{i}}}\left(\begin{array}{cc}
\sqrt{\lambda} D_{x}^{i} & \sqrt{\lambda} D_{y}^{i} \\
\sqrt{\mu} D_{y}^{i} & \sqrt{\mu} D_{x}^{i} \\
\sqrt{2 \mu} D_{x}^{i} & 0 \\
0 & \sqrt{2 \mu} D_{y}^{i}
\end{array}\right) \phi^{i}\right\|^{2} .
\end{aligned}
$$

where $K$ is given by

$K=\left(\begin{array}{cc}(\lambda+2 \mu) D_{x}^{i t} D_{x}^{i}+\mu D_{y}^{i t} D_{y} & \lambda D_{x}^{i t} D_{y}+\mu D_{y}^{i t} D_{x}^{i} \\ \lambda D_{y}^{i t} D_{x}^{i}+\mu D_{x}^{i t} D_{y}^{i} & (\lambda+2 \mu) D_{y}^{i t} D_{y}^{i}+\mu D_{x}^{i t} D_{x}^{i}\end{array}\right)$

This method is used to discretize both $E\left(\phi_{S}\right)$ and $E\left(\phi_{A}\right)$. It can be seen from (13) and (3) that the cost function is quadratic. We minimize (3) with respect to both $\phi_{S}$ and $\phi_{A}$, with the corpus callosum fixed at the boundary of the unit square, to compute the sulcally-coregistered flat maps for both brains simultaneously. The minimization is performed by using a preconditioned conjugate gradient method with Jacobi preconditioner. In practice the minimization algorithm converges in approximately 500 iterations, requiring 3-4 mins on a desktop computer for surfaces with approximately 200,000 vertices.

\section{RESULTS AND VALIDATION}

We first extract cortical surfaces from MRI for each subject using the BrainSuite software [20] to produce a genus-zero tessellated representation of the inner gray/white cortical boundary. We then manually delineate 23 major sulci on each of these extracted cortical hemisphere meshes. Delineation is performed in accordance with a sulcal labeling protocol with established intra- and inter- rater reliability [3]. This protocol specifies that sulci do not intersect and that individual sulci are continuous curves that are not interrupted. If interruptions are present the curves are simply interpolated across any interrupting gyri. In cases where a full set cannot be defined, a subset can be used without any change in the algorithm defined here. Uniform samples along the sulcal curves serve as landmarks in our registration. Fig. 1 illustrates the alignment process. Fig. 2 shows the RMS error in matching of sulcal landmarks and the percentage area of overlap or folding in the flat maps as a function of the Lagrange multiplier $\sigma$. Enforcing a more accurate sulcal alignment by increasing $\sigma$ results in an increase in overlap in the mappings. We choose $\sigma=3$ for further analysis. Although the elastic mappings are not formally guaranteed to produce a bijective registration, we found that by setting $\mu=1, \lambda=10$ and $\sigma=3$, we can achieve a nearly bijective map with an average overlap of approximately $0.4 \%$ of the surface area. By inspection we see that the overlap occurs in the vicinity of pairs of landmarks that are closely spaced in one brain and distant in the

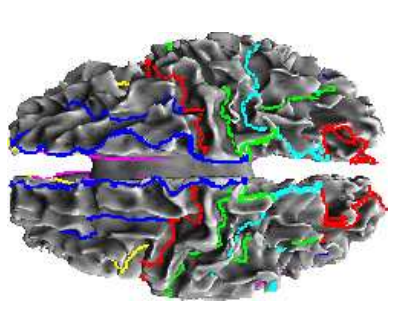

(a) Surface 1

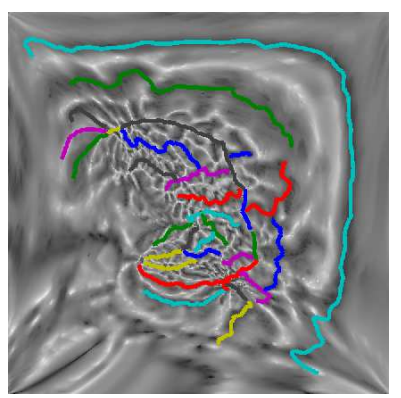

(c) $\sigma=0$ for surface 1

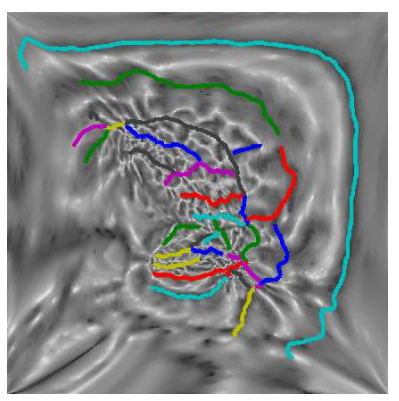

(e) $\sigma=3$ for surface 1

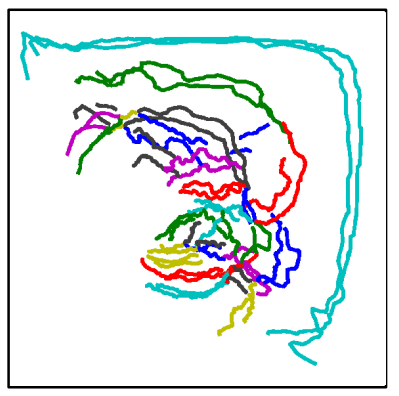

(g) Sulcal alignment for $\sigma=0$

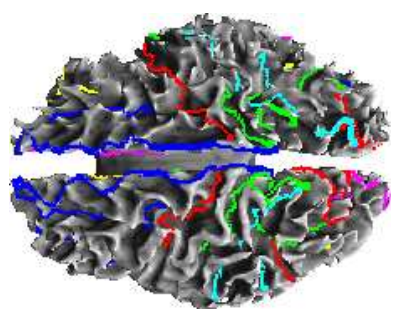

(b) Surface 2

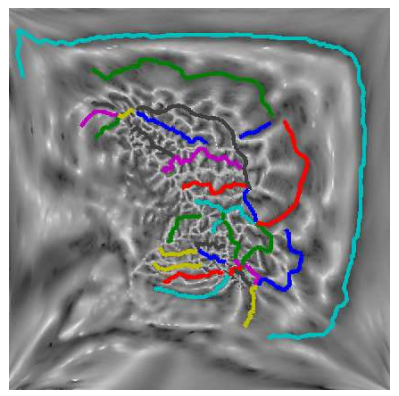

(d) $\sigma=0$ for surface 2

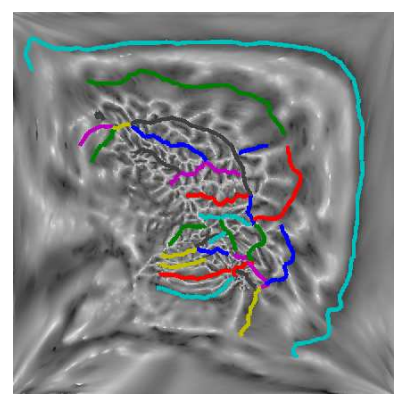

(f) $\sigma=3$ for surface 2

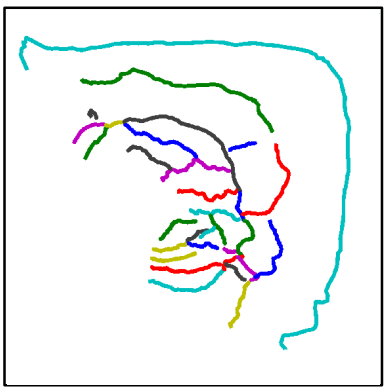

(h) Sulcal alignment for $\sigma=3$
Fig. 1. (a),(b) The two cortical surfaces with hand labeled sulci as colored curves; (c),(d) flat maps of a single hemisphere for the two brains without the sulcal alignment constraint; (e),(f) flat maps with sulcal alignment; (g),(h) overlay of sulcal curves on the flat maps, without and with alignment, respectively. 


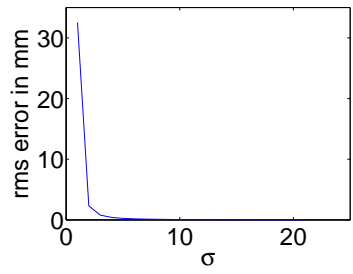

(a) RMS error as a function of $\sigma$

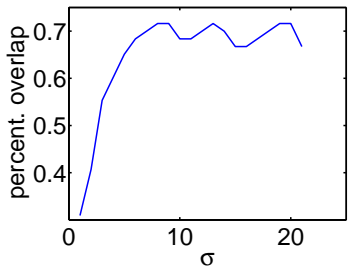

(b) Percentage overlap
Fig. 2. RMS error and percentage overlap in the flattened map as a function of $\sigma$.

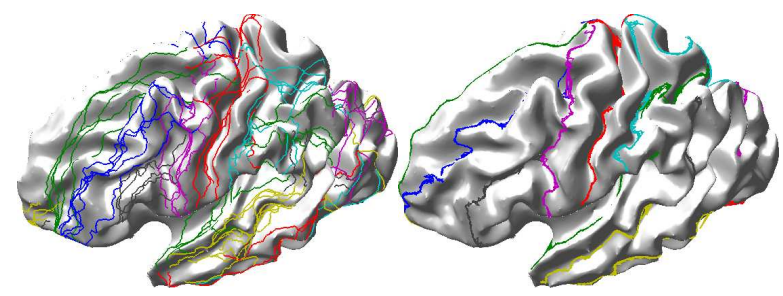

Fig. 3. Mapping of sulcal landmarks from 5 subjects to the atlas brain (left) without and (right) with the sulcal alignment constraint.

other. One solution to this problem is to locally reparameterize in the neighborhood of the overlap once the flat maps are computed.

We performed a leave-one-out validation for examining the performance of our method. We choose one brain as a representative 'atlas' and align cortices of 5 subjects with the atlas using 22 of the 23 labeled sulci leaving one sulcus out of the registration each time. For each of the registrations, we measured how well the sulcus that was left out of the registration process aligned across the subjects with $(\sigma=0)$ and without $(\sigma=3)$ sulcal alignment. Without alignment, there was an RMS error of $33.1 \mathrm{~mm}$ in the free sulcus. With alignment using all but the free sulcus, the remaining rms error was $3.2 \mathrm{~mm}$ for the free sulcus.

Incorporating sulcal landmark alignment directly in our parameterization method not only avoids the resampling and reparameterization steps and reduces computational cost while maintaining high resolution in the surface tessellations, but also makes the registration inverse consistent. The improved speed and resolution of the registration may help in large scale and detailed comparisons of cortical data.

\section{REFERENCES}

[1] P. M. Thompson, M. S. Mega, C. Vidal, J. Rapoport, and A. W. Toga, "Detecting disease-specific patterns of brain structure using cortical pattern matching and a population-based probabilistic brain atlas," in IPMI, 2001, pp. 488-501.

[2] N. Gogtay et al., "Dynamic mapping of human cortical development during childhood through early adulthood," PNAS, vol. 101, pp. 8174-8179, 2004.
[3] P. M. Thompson et al., "Detecting dynamic and genetic effects on brain structure using high dimensional cortical pattern matching," in Proc. ISBI, 2002.

[4] G. G. Nahas and T. F. Burks, Eds., Drug Abuse in the Decade of the Brain, IOS Press, 1997.

[5] P. Thompson et al., "Structural abnormalities in the brains of human subjects who use methamphetamine," Journal of Neuroscience, vol. 24, no. 26, pp. 6028-6036, 2004.

[6] M. K. Hurdal et al., "Coordinate system for conformal cerebellar flat maps," NeuroImage, vol. 11, pp. S467, 2000.

[7] M. Bakircioglu, U. Grenander, N. Khaneja, and M. I. Miller, "Curve matching on brain surfaces using frenet distances," $\mathrm{Hu}$ man Brain Mapping, vol. 6, pp. 329-333, 1998.

[8] B. Fischl et al., "High-resolution inter-subject averaging and a coordinate system for the cortical surface," Human Brain Mapping, vol. 8, pp. 272-284, 1998.

[9] D. Tosun, M. E. Rettmann, and J. L. Prince, "Mapping techniques for aligning sulci across multiple brains," Medical Image Analysis, vol. 8, no. 3, pp. 295-309, 2005.

[10] P. M. Thompson el al., "Mapping cortical change in Alzheimer's disease, brain development and schizophrenia," NeuroImage, vol. 23, no. 1, pp. S2-S18, Sept. 2004.

[11] Y. Wang, X. Gu, K.M. Hayashi, T.F. Chan, P.M. Thompson, and S.T. Yau, "Brain surface parameterization using Riemann surface structure," in MICCAI 2005, 2005, pp. 657-665.

[12] A. A. Joshi, D. W. Shattuck, P. M. Thompson, and R. M. Leahy, "A framework for registration, statistical characterization and classification of cortically constrained functional imaging data," in LNCS, July 2005, vol. 3565, pp. 186-196.

[13] H. J. Johnson and G. E. Christensen, "Consistent landmark and intensity-based image registration," IEEE Transactions on Medical Imaging, vol. 21, no. 5, pp. 450-461, 2002.

[14] S. Angenent, S. Haker, A. Tannenbaum, and R. Kikinis, "Laplace-Beltrami operator and brain surface flattening," IEEE TMI, vol. 18, pp. 700-711, 1999.

[15] B. Tang, G. Sapiro, and V. Caselles, "Diffusion of general data on non-flat manifolds via harmonic maps theory: The direction diffusion case.," International Journal of Computer Vision, vol. 36, no. 2, pp. 149-161, 2000.

[16] A. A. Joshi, R. M. Leahy, P. M. Thompson, and D. W. Shattuck, "Cortical surface parameterization by $p$-harmonic energy minimization.," in ISBI, 2004, pp. 428-431.

[17] Y. Wang, L. M. Lui, T. F. Chan, and P. M. Thompson, "Optimization of brain conformal mapping with landmarks," in MICCAI 2005: Proceedings, Part II, 2005, pp. 675-683.

[18] D. W. Shattuck and R. M. Leahy, "BrainSuite: An automated cortical surface identification tool.," in MICCAI, 2000, pp. 5061.

[19] G. Hermosillo, C. Chefd'Hotel, and O. Faugeras, "Variational methods for multimodal image matching," International Journal of Computer Vision, vol. 50, no. 3, pp. 329-343, Dec. 2002.

[20] D. W. Shattuck and R. M. Leahy, "BrainSuite: An automated cortical surface identification tool," Medical Image Analysis, vol. 8, no. 2, pp. 129-142, 2002. 